\title{
Self-Evaluation in a Clinical Setting to Develop Nursing Students' Clinical Judgment
}

\author{
Tanya Sobaski $^{1} \&$ Sam Abraham ${ }^{1}$ \\ ${ }^{1}$ Bethel College School of Nursing, Mishawaka, Indiana, USA \\ Correspondence: Sam Abraham, Associate Professor of Nursing, 1001 Bethel Circle, Bethel College School of \\ Nursing, Mishawaka, Indiana, 46545, USA.
}

Received: November 15, 2017

Accepted: December 3, 2017 Online Published: December 8, 2017

doi:10.20849/ijsn.v3i1.241

URL: https://doi.org/10.20849/ijsn.v3i1.241

\begin{abstract}
Background: The Lasater clinical judgment rubric is based upon Tanner's clinical judgment model for developing clinical judgment and the incorporation of the Benner Novice to Expert theory. The Lasater clinical judgment rubric has been used in nursing programs at the baccalaureate level and with simulation exercises. Method: In this study, the Lasater clinical judgment rubric was used to compare instructor and associate degree nursing students' evaluations in an acute care setting during their first nursing care rotation. Data analysis included a split-plot ANOVA with repeat measures. A sample size of 16 students yielded an effect size of .40 with $\alpha=.001$.

Results: There was no significant difference in mean scores between the five administrations of the assessment with different groups.

Conclusion: The interactions between the evaluator and the scores over time were consistent between groups. Development of student's ability to use self-evaluation, introspection, and self-awareness skills are foundational for thinking that is more complex.
\end{abstract}

Keywords: nursing education, clinical judgment, performance based self-evaluation, Lasater clinical judgment rubric

\section{Introduction}

The nursing student in the clinical setting may experience situations that challenge theoretical classroom learning. Students are excited to apply their learning by providing care to patients. Achieving the full value of the educational experience, the student must participate in evaluating their experience. "Behaviors that facilitate learning and encourage reflection, and various initiatives promote innovation and individualization for future nurses" (Papathanasiou, Konstantinos, \& Sarafis, 2014, p. 57). Evaluation of one's experiences develops with introspection and translation of the experience combined with the theoretical knowledge through application. "Clinical judgment has been identified as a critical component of professional nursing practice and enables nurses to deliver safe patient care with optimal outcomes" (Miraglia \& Asselin, 2015, p. 284) It is through purposeful reflection and self-evaluation about personal performance that the development of clinical judgment skills can occur.

Discovery of the possibilities through self-awareness of the situation is a precursor to responding with critical thinking and reasoning. Based upon the outcome of the situation and the feedback received, from the instructor, nursing staff, and peers, the student's self-efficacy will be either reinforced or weakened. "Nursing students perceive availability, approachability and feedback from the clinical facilitator to be highly influential to their learning in the clinical setting" (Sweet \& Broadbent, 2017, p. 30). This pilot study used the Lasater clinical judgement rubric (LCJR) along with Benner's theory of Novice to Expert to heighten self-awareness and self-evaluation of experiences in relationship to patient care and clinical performance. Nielsen, Lasater, and Stock (2016) emphasized that a structured framework provides objective ways to evaluate and aid develop clinical judgment.

Emerson (2007) discussed tips for effectively appraising student performance including self-evaluation and suggestions for how evaluations are shared. Learning in the clinical setting is facilitated with the guidance of an effective instructor. Carwile and Murrell (2002) affirmed, through self-evaluation, students may experience 
increased satisfaction with the educational process and decreased anxiety during evaluation (p. 415). Boyer, Tardif, and Lefebvre (2015) in a study on how nursing students think in clinical situations indicated three levels of development in clinical judgment in undergraduate nursing students; (1) intervening step-by-step, where the student follows procedures and is focused on the patient's health problem; (2) intervening to understand, where the student applies reasoning process and is focused on analyzing the health problem; (3) responding to the whole person, where the student integrates combined influences and is focused on the health experiences (p. 627).

The LCJR focuses on concepts of noticing, interpreting, responding and reflecting with different levels of achievement based upon common, clear terminology. Using a self-evaluation tool, such as the LCJR, serves to guide the faculty and student with varying levels of development in critical thinking.

\section{Identifying Assumptions}

Benner's theory of novice to expert has "three general aspects of skilled performance" (Benner, 1984, p. 13). The aspects are described as a development of skill and knowledge as the student's focus progresses from a concrete to abstract level of thinking, leading to an understanding of information relevancy, and then from an observatory to a participatory role (Benner, 1984, p. 13). It is through reflection upon lived experiences that true learning occurs. Shifting the evaluative process from the instructor to the student will assist the student to develop a deeper level of thinking.

Tanner's clinical judgment model "provides guidance for faculty members to help student's diagnosis breakdowns, identify areas needed for growth, and consider learning experiences that focus attention on those areas" (Tanner, 2006, p. 208). The Tanner model has four major aspects of noticing, interpreting, responding, and reflecting, that "provide language to describe how nurses think when they are engaged in complex, undetermined clinical situations that require judgment" (Tanner, 2006, p. 209). Use of the model may help faculty guide students' learning and help students in developing their clinical judgment and reasoning skills (Nielsen et al., 2007; Tanner, 2006).

\section{Research Questions}

RQ1. Is the Lasater clinical judgment rubric (LCJR) a reliable evaluation tool in the associate level nursing program?

RQ2. Will using the LCJR in the first semester of the nursing program assist in development of students' clinical judgment in the clinical setting?

\section{Methods and Procedures}

The initiation of the student self-evaluation took place in the first semester of the first year in an associate level-nursing program. This was the students' first clinical experience outside of the clinical lab. One instructor for two groups was the evaluator for consistency of evaluation and reliability of the rubric. The instructor was an individual independent of the sole investigator. The instructor selected had more than 20 years of experience teaching at this level and was the only instructor used for both rotations.

\section{Population and Sample}

First-year, first-semester students in the Associate Degree of Nursing were included in this study. The students in adult clinical rotations were assigned to an acute care unit, bi-weekly, with two separate, but consecutive seven-week rotations. Exclusion of participants were students in clinical groups assigned to different acute or long term care settings, having different clinical instructors, and not being a first-year nursing student. Sample size was eight students per rotation, yielding 16 students between the two clinical groups. Convenience sampling was chosen to provide continuity to the current research. For this research, two groups were used at the same facility for different rotations.

\section{Research Design}

Using a quasi-experimental longitudinal design with the LCJR and scoring scale repeated measures testing occurred nine times. The first group was tested four times and the second group was tested five times. The range of possible scores is 1-4, with four as the highest score possible and one is the lowest score possible for each area of the rubric. The scoring is as follows: 1 (Beginning), 2 (Developing), 3 (Accomplished), and 4 (Exemplary). Lasater (2007) reported a continuation of "predictive validity studies formalizing the correlation between the simulation laboratory and clinical setting, and studies of interrater reliability" (p. 503). Adamson et al. (2014) has also established the reliability and validity of the LCJR with simulation using standardized human patients (Adamson et al., 2012). 


\section{Ethical and Legal Considerations}

This study was granted Institutional Review Board (IRB) approval by the investigator's college and the IRB of the hospital participating in the study. The research participants received and signed an informed consent for the LCJR study.

\section{Implementation Protocol}

Repeated instruction of the LCJR and the scoring sheet was provided by the investigator and presented prior to each clinical groups' orientation. The student's decision to participate in this study was voluntary, however all students chose to participate. Students were informed that they could choose to end their participation at any time during the study. Participation in the study had no effect on the student's grade positively or negatively. Daily clinical evaluations are formative, therefore there was no direct effect on a student's grades for the clinical portion.

\section{Data Collection Process}

The evaluation scoring sheets were collected with the student and instructor evaluations placed in separate envelopes. The investigator collected the envelopes from a designated student and from the instructor separately. Data entry was completed weekly and entered into an Excel file. The researcher made back-up copies of electronic files to a flash drive and external hard drive.

\section{Results}

WINKS Statistical Analysis Data Software 6.0.91 Professional Edition was used to analyze a mixed design ANOVA with repeated measures for each of the 11 traits and subscales of the LCJR. The 11 different traits are Focused Observation (FO), Recognizing Deviations from Expected Patterns (RDEP), Information Seeking (IS); Prioritizing Data (PD), Making Sense of Data (MSD), Calm, Confident Manner (CCM), Clear Communication (CC), Well Planned Interventions/ Flexibility (WPI/F), Being Skillful (BS), Evaluation/Self-Analysis (E/SA), and Commitment to Improvement (CI). The data analysis provided the following information. There was found to be no significant difference in means of the students' self-evaluation and the instructor's scores. There was no significant difference in mean scores between the five administrations of the assessment. There was consistency in the interaction between the evaluator and the scores over time. The 11 subscales (traits) compared for cell means and are displayed individually in table formats (see Tables 1-11).

Table 1. Focused observation

\begin{tabular}{lll}
\hline Occurrence & Instructor's Mean Score & Student's Mean Score \\
\hline 1 & $1.0^{*}$ & $2.7^{* *}$ \\
2 & $2.3^{*}$ & $2.7^{* *}$ \\
3 & $2.1^{*}$ & $2.7^{* *}$ \\
4 & $2.1^{*}$ & $2.8^{* *}$ \\
5 & $2.6^{*}$ & $3.0^{* *}$ \\
\hline
\end{tabular}

Note. Focused Observation (FO) is the ability to recognize object and subjective data and prioritization of the meaning of the information. The mean for the instructor's first assessment improved over time with repeated measures to the last assessment, which indicated no significant difference in the scores over time. This indicates a significant interaction between the instructor and students' evaluations over time. The greatest significance occurred with the initial interaction between the instructor and students' evaluations.

$* \mathrm{p}<.001 . * * \mathrm{p}=.848$.

Table 2. Recognizing deviations from expected patterns

\begin{tabular}{lll}
\hline Occurrence & Instructor's Mean Score & Student Mean Score \\
\hline 1 & $1.0^{*}$ & $2.5^{* *}$ \\
2 & $2.3^{*}$ & $2.5^{* *}$ \\
3 & $2.0^{*}$ & $2.8^{* *}$ \\
4 & $2.1^{*}$ & $2.7^{* *}$ \\
5 & $2.4^{*}$ & $2.8^{* *}$ \\
\hline
\end{tabular}

Note. Recognizing Deviations from Expected Patterns (RDEP) analyzed patterns and meaning of variations related to assessment findings. This was a significant result but only for the first interaction between the instructor evaluations and the students' self-evaluations.

$* \mathrm{p}<.001 . * * \mathrm{p}=.65$. 
Table 3. Information seeking

\begin{tabular}{lll}
\hline Occurrence & Instructor's Mean Score & Student Mean Score \\
\hline 1 & $1.0^{*}$ & $2.8^{* *}$ \\
2 & $2.4^{*}$ & $2.6^{* *}$ \\
3 & $2.4^{*}$ & $3.0^{* *}$ \\
4 & $2.6^{*}$ & $2.8^{* *}$ \\
5 & $2.6^{*}$ & $3.2^{* *}$ \\
\hline
\end{tabular}

Note. The significant difference was between the instructor and students' interaction with the initial evaluation; however, the data indicated that there was no significant difference in the assessment results.

$* \mathrm{p}<.001 .{ }^{* *} \mathrm{p}=.589$.

Table 4. Prioritizing data

\begin{tabular}{lll}
\hline Occurrence & Instructor's Mean Score & Student Mean Score \\
\hline 1 & $1.0^{*}$ & $2.3^{* *}$ \\
2 & $2.4^{*}$ & $2.3^{* *}$ \\
3 & $2.1^{*}$ & $2.5^{* *}$ \\
4 & $2.0^{*}$ & $2.5^{* *}$ \\
5 & $2.1^{*}$ & $2.8^{* *}$ \\
\hline
\end{tabular}

Note. Prioritizing Data (PD) involved the ability to interpret information regarding a patient's situation. The difference was significant between the instructor and students with the initial interaction.

$* \mathrm{p}<.001 . * * \mathrm{p}=.639$.

Table 5. Making sense of data

\begin{tabular}{lll}
\hline Occurrence & Instructor's Mean Score & Student Mean Score \\
\hline 1 & $1.0^{*}$ & $2.5^{* *}$ \\
2 & $2.0^{*}$ & $2.3^{* *}$ \\
3 & $2.0^{*}$ & $3.0^{* *}$ \\
4 & $2.0^{*}$ & $2.5^{* *}$ \\
5 & $2.1^{*}$ & $2.7^{* *}$ \\
\hline
\end{tabular}

Note. Making Sense of Data (MSD) was the capacity to understand data to sense the nuisances of patterns and implement actions to improve patient outcomes.

There was a significant difference in the interaction between subjects and over time.

$* \mathrm{p}<.001 . * * \mathrm{p}=.054$.

Table 6. Calm, confident manner

\begin{tabular}{lll}
\hline Occurrence & Instructor's Mean Score & Student Mean Score \\
\hline 1 & $1.0^{*}$ & $2.8^{* *}$ \\
2 & $2.3^{*}$ & $3.0^{* *}$ \\
3 & $2.4^{*}$ & $2.7^{* *}$ \\
4 & $2.3^{*}$ & $2.7^{* *}$ \\
5 & $2.4^{*}$ & $2.7^{* *}$ \\
\hline
\end{tabular}

Note. The ability to respond to leadership and ability to delegate in stressful situations is what the LCJR refers to as Calm, Confident Manner (CCM). This signified a significant difference of the self-evaluations, with interaction between the instructor and students at the initial test.

$* \mathrm{p}<.001 . * * \mathrm{p}=.718$ 
Table 7. Clear communication

\begin{tabular}{lll}
\hline Occurrence & Instructor's Mean Score & Student Mean Score \\
\hline 1 & $1.3^{*}$ & $2.7^{* *}$ \\
2 & $2.4^{*}$ & $3.0^{* *}$ \\
3 & $2.4^{*}$ & $2.7^{* *}$ \\
4 & $2.5^{*}$ & $2.7^{* *}$ \\
5 & $2.4^{*}$ & $3.0^{* *}$ \\
\hline
\end{tabular}

Note. Clear Communication (CC), reflects the effective communication using therapeutic techniques, involvement of the healthcare team, and verification of understanding of instruction and dialogue. The results indicate a significant difference in interaction between the instructor evaluations and the students' evaluations over time, with the greatest significance at the initial interaction.

$* \mathrm{p}<.001 . * * \mathrm{p}=.650$.

Table 8. Well planned interventions and flexibility

\begin{tabular}{lll}
\hline Occurrence & Instructor's Mean Score & Student Mean Score \\
\hline 1 & $1.1^{*}$ & $2.5^{* *}$ \\
2 & $2.3^{*}$ & $2.5^{* *}$ \\
3 & $2.3^{*}$ & $2.3^{* *}$ \\
4 & $2.6^{*}$ & $2.2^{* *}$ \\
5 & $2.7^{*}$ & $2.7^{* *}$ \\
\hline
\end{tabular}

Note. Well Planned Interventions and Flexibility (WPI/F) is described as individualization of patient -specific interventions and adjustment of interventions based upon the patient's response. The results indicated a significant difference with the interaction of instructor evaluation and the students' self-evaluation.

$* \mathrm{p}<.001 . * * \mathrm{p}=.662$

Table 9. Being skillful

\begin{tabular}{lll}
\hline Occurrence & Instructor's Mean Score & Student Mean Score \\
\hline 1 & $1.1^{*}$ & $2.5^{* *}$ \\
2 & $2.3^{*}$ & $2.5^{* *}$ \\
3 & $2.3^{*}$ & $2.3^{* *}$ \\
4 & $2.6^{*}$ & $2.2^{* *}$ \\
5 & $2.7^{*}$ & $2.7^{* *}$ \\
\hline
\end{tabular}

Note. The instructor and students evaluated Being Skillful (BS) during the course of this study. Being Skillful is level of competence in completing nursing skills. There was a significant difference with the initial interaction between the instructor and student self-evaluations.

$* \mathrm{p}<.001 . * * \mathrm{p}=.431$.

Table 10. Evaluation/self-analysis

\begin{tabular}{lll}
\hline Occurrence & Instructor's Mean Score & Student Mean Score \\
\hline 1 & $1.1^{*}$ & $2.5^{* *}$ \\
2 & $2.3^{*}$ & $2.5^{* *}$ \\
3 & $2.3^{*}$ & $2.3^{* *}$ \\
4 & $2.6^{*}$ & $2.2^{* *}$ \\
5 & $2.7^{*}$ & $2.7^{* *}$ \\
\hline
\end{tabular}

Note. Evaluation/Self-Analysis (E/SA) is critically analyzing personal performance and appraisal of choices. There was a significant difference with interaction between the initial instructor and students' scores and successive scores.

$* \mathrm{p}<.001 .{ }^{* *} \mathrm{p}=.573$. 
Table 11. Commitment to improvement

\begin{tabular}{lll}
\hline Occurrence & Instructor's Mean Score & Student Mean Score \\
\hline 1 & $1.0^{*}$ & $2.7^{* *}$ \\
2 & $2.3^{*}$ & $2.8^{* *}$ \\
3 & $2.6^{*}$ & $2.7^{* *}$ \\
4 & $2.7^{*}$ & $2.5^{* *}$ \\
5 & $2.9^{*}$ & $2.8^{* *}$ \\
\hline
\end{tabular}

Note. Commitment to Improvement (CI) is the continual commitment to personal process improvement and identification of strengths and weaknesses. There was a significant difference in the interaction between the instructor and students' evaluations initially.

$* \mathrm{p}=.012 . * * \mathrm{p}=.146$.

\section{Data Analysis Procedures}

Data analysis using a two-way ANOVA with repeated measures testing for mean differences between two or more independent groups was conducted using WINKS Statistical Analysis Data Software 6.0.91 Professional Edition. A comparison of cell means was performed instead of a marginal means comparison, due to the significant effect between the first interaction and the remaining interactions as indicated by the result of the third hypothesis.

A mixed design ANOVA with repeated measures was conducted for the subscale[s] of the LCJR utilizing the WINKS Statistical Analysis Data Software 6.0.91 Professional Edition. There was significant interaction effect between the main effects of the evaluator and repeated measures over time $(F(4,40)=4.31, p<.005)$.

Power analysis using the Newman-Keuls post hoc test with the level of significance at $\alpha=0.001$ reduced "Type 1 error." The Newman-Keuls test makes comparisons based upon groups to compensate for repeated t-tests. The sample size of 16 students with repeated measures testing 5 times, would theoretically "provide over 500 paired-wise comparisons," leading to a normal probability distribution and yield a large effect size of 0.40 . Repeated measures testing controlled the $\beta$ type II error.

\section{Discussion}

The evaluation scores were calculated comparing the students' and instructor's responses over two clinical rotations in the hospital setting and the progression of clinical judgment scores utilizing the LCJR as the measurement tool. Analyzing the interaction of students over time demonstrated no significant effect $\mathrm{f}=4.31$; however, there was a significant effect with students and instructor scores between the first evaluation and subsequent evaluations $\mathrm{f}=4.25$. After the first evaluation, no significant difference was found in the evaluations between the students and instructor.

Table 12. Interaction means over time

\begin{tabular}{lll}
\hline Evaluation \# & Instructor Mean & Student Mean \\
\hline 1 & 1.0 & 2.5 \\
2 & 2.2 & 2.5 \\
3 & 2.5 & 2.7 \\
4 & 2.3 & 2.8 \\
5 & 2.8 & 2.8 \\
\hline
\end{tabular}

Note. Results revealed that the major difference occurred with the first measurement of the students' self-evaluation and subsequent evaluations. The final overall evaluation results were statistically the same.

Possibilities for the initial difference in the scoring could be the students' lack of understanding in how to utilize the evaluation tool or students' inflated perception of competency in clinical proficiency. It is possible that after the first experience, completing the LCJR, and having time to reflect upon their level of achievement, students were able to improve their accuracy in self-evaluation. Another possibility is the students having a distorted view of their abilities with the first evaluation. Papathanasiou et al. (2014) found that "there was a disparity in expectations and the reality of the clinical learning environment for students in nursing" (p. 57).

This being the students' first rotation of nursing school, they did not have a reference point for clinical evaluation, 
or the skill set for self-evaluation, whereas the instructor had a reference point for evaluating students. After the initial assessment the evaluations were statistically the same $p=.005$. The instructor, independent of the self-evaluations completed, gave students written formative evaluations. This feedback allowed students to correct their thought processes and improve their reliability in their expectations and self-evaluation. The argument can be made that after the initial calibration the LCJR was reliable for the students and between the instructor and students.

\section{Limitations}

Limitations of this study include the ambiguity of the clinical setting, the study focused on first year, first semester students that may have had unrealistic expectations and perceptions of their abilities and effectiveness. Other limitations were occasional incomplete scoring sheets by students and the students' lack of familiarity with the self-evaluation process and rubric used. It is also possible that students inflated their scores in the self-evaluation, believing that higher scores would influence the instructor to score the students' evaluation higher. Another limitation is the small sample size.

\section{Conclusion}

The process of developing effective self-evaluation, incorporating Benner's theory of knowledge acquisition through experiential learning (Benner, 1984), Tanner's conceptual clinical judgment model integrating constructs of noticing, reflecting, responding, and interpreting, and the LCJR to achieve effective self-evaluation (Tanner, 2006; Lasater, 2007) has been demonstrated through the results of this study. The students' adjustment of their self-evaluation scores and the alignment of the results with the instructor's scores after the initial evaluation, demonstrate the LCJR is an accurate tool for self-evaluation. The implementation of the LCJR in the first year associate's level nursing program assisted students in developing self-evaluation skills by increasing introspection and self-awareness. Faculties continue to evaluate students to determine clinical objective attainment; however, with the LCJR, students would continue the learning process, as they re-think their clinical experiences. Based on the findings in this study, the LCJR is an effective tool to help students learn to adjust to the expectations of their instructor and to develop a realistic viewpoint of their abilities in the clinical setting.

\section{Acknowledgement}

The authors wish to acknowledge late Dr. Karon Schwartz, Bethel College, Indiana, for offering valuable comments during the early stages of the study. We also like to thank Bethel College School of Nursing and Lakeland HealthCare administrators for allowing the opportunity to conduct this study.

\section{References}

Adamson, K. A., Gubrud, P., Sideras, S., \& Lasater, K. (2012). Assessing the reliability, validity, and use of the Lasater Clinical Judgement Rubric: three approaches. Journal of Nursing Education, 51(2), 66-73. https://doi.org/10.3928/01484834-20111130-03

Benner, P. (1984). From novice to expert: Excellence and power in clinical nursing practice. Menlo Park, CA: Addison-Wesley.

Boyer, L., Tardif, J., \& Lefebvre, H. (2015). From a medical problem to a health experience: How nursing students think in clinical situations. Journal of Nursing Education, 54(11), 625-632. http://dx.doi.org/10.3928/01484834-20151016-03

Carwile, L., \& Murrell, J. (2002). Student self-evaluation in clinical education. Radiologic Technology, 73(5), 415-422.

Emerson, R. J. (2007). Nursing education in the clinical setting. Elsevier-Mosby.

Lasater, K. (2007). Clinical judgment development: Using simulation to create an assessment rubric. Journal of Nursing Education, 46, 496-503.

Lasater, K., \& Nielsen, A. (2009a). The influence of concept-based learning activities on student's clinical judgment development. Journal of Nursing Education, 48, 441-446. https://doi.org/10.3928/014848434-20090717-03

Miraglia, R., \& Asselin, M. (2015). The Lasater Clinical Judgment Rubric as a framework to enhance clinical judgment in novice and experienced nurses, J. Nurses Prof. Dev., 31(5), 284-291. https://doi.org/10.1097/NND.0000000000000209

Nielsen, A. (2009). Concept-based learning activities using the clinical judgment model as a foundation for clinical learning. Journal of Nursing Education, 48, 353-35. 
https://doi.org/10.9999/01484834-20090515-09

Nielsen, A., Lasater, K., \& Stock, M. (2016). A framework to support preceptors' evaluation and development of new nurses' clinical judgment. Nurse Education in Practice, 19, 84-90. http://dx.doi.org/10.1016/j.nepr.2016.03.012

Papathanasiou, I. V., Konstantinos, T., \& Sarafis, P. (2014). Views and perceptions of nursing students on their clinical learning environment: Teaching and learning. Nurse Education Today, 34(1), 57-60. https://doi.org/10.1016/j.nedt.2013.02.007

Sweet, L., \& Broadbent, J. (2017). Nursing students' perceptions of the qualities of a clinical facilitator that enhance learning. Nurse Education in Practice, 22, 30-36. http://dx.doi.org/10.1016/j.nepr.2016.11.007

Tanner, C. A. (2006). Thinking like a nurse: A research-based model of clinical judgment in nursing. Journal of Nursing Education, 45, 204-211.

\section{Copyrights}

Copyright for this article is retained by the author(s), with first publication rights granted to the journal.

This is an open-access article distributed under the terms and conditions of the Creative Commons Attribution license (http://creativecommons.org/licenses/by/4.0/). 